\title{
Antenatal and Postnatal Experiences of Women during Covid-19 Pandemic: The Past Meets the Present
}

\author{
Alara Barnes* and Prof. Jennifer Kelly, PhD \\ School of Health and Biomedical Sciences, RMIT University, Victoria, Australia
}

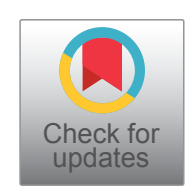

\begin{abstract}
Coronavirus disease 19 (COVID-19) is an extremely infectious viral illness that emerged as a world-wide health issue in early 2020. The long-term impact of COVID-19 and the imposed measures to reduce transmission are not fully recognised. More specifically, the effect of COVID-19 on the experiences of pre and postnatal women during this pandemic is not fully understood despite being vital to addressing potential needs of women, infants and families.

Historically, epidemics and pandemics have had significant impact on the physical and psychological health of women who are pregnant. The COVID-19 pandemic will potentially affect the health and wellbeing of individuals as a result of the infection as well as the measures imposed to reduce transmission. Research is starting to emerge regarding the impact of COVID-19 on the mental health of women who were pregnant and give during the pandemic. However, there is limited literature and limited qualitative research that has focused on the antenatal and postnatal women in terms of physical and psychosocial issues.

Recent studies have concluded that mechanisms to control COVID-19 such as lockdown measures, isolating from others and quarantine have led to a substantial burden on individuals. The need for further research into the mental well-being of people during COVID-19 is an international health priority. This exploration of the literature illustrates the significance of conducting a qualitative study into the pre and postnatal experiences of pregnant women during the COVID-19 pandemic.
\end{abstract}

Keywords

Covid-19, Pre and postnatal women, Pandemics and epidemics, Psychological adjustments, Pregnancy, Birth

\section{Introduction}

Coronavirus disease 19 (COVID-19) emerged as a worldwide health issue in early 2020 although the timing of the first reported case remains unclear as is the spread of the disease out of China. For instance, the World Health Organisation investigation revealed the source and origin continues to be unknown [1]. As such, the long-term impact of COVID-19 and the imposed measures to reduce transmission are not fully recognised. More specifically, the effect of COVID-19 on the experiences of pre and postnatal women during this pandemic is not fully understood despite being vital to understanding potential needs of women, infants and families [2].

This paper will explore the impact of past pandemics and epidemics on the physical, psychosocial and mental health experiences of women who were pregnant to provide an historical perspective to the current pandemic. Additionally, this paper will explore the key psychological adjustments that characteristically occur in pregnancy to afford context to this study into the antenatal and postnatal experiences of women during COVID-19. Further, this paper will focus on exploring the impact of COVID-19 on the psychological and social wellbeing of women who are pregnant and birth during the pandemic.

\section{Historical Context: Past Pandemics}

Pregnancy is deemed an exciting journey for most women, typically filled with happiness and anticipation, with families eager to meet the new child. However, this experience may be significantly altered for women who are pregnant and birth during a global pandemic or epidemic. Throughout history there have been various pandemics and epidemics that have altered the experiences and health outcomes of pregnant women and their children, with key examples being Ebola virus, human immunodeficiency virus (HIV) and acquired immunodeficiency syndrome (AIDS), and hemagglutinin type 1 and neuraminidase type 1 (H1N1) influenza. For the pur-

\footnotetext{
*Corresponding author: Dr. Alara Barnes, School of Health and Biomedical Sciences, RMIT University, 9 Wonkana Road Glenfield Park NSW 2650, Victoria, Australia, Tel: 0492811055

Accepted: June 09, 2021

Published online: June 11, 2021

Citation: Barnes A, Kelly J (2021) Antenatal and Postnatal Experiences of Women during Covid-19 Pandemic: The Past Meets the Present. J Nurs Pract 4(1):302-308
} 
pose of this paper, a pandemic is an epidemic that has spread worldwide, with case numbers far greater than typically reported $[3,4]$. An epidemic is defined as a disease that occurs suddenly and effects communities, regions or a country in excess numbers $[3,4]$. One of the most eminent epidemics to have occurred to date is Ebola Virus.

\section{Ebola virus}

The Ebola virus outbreak in West Africa between 2014 and 2016 killed at least 11,300 people and caused extreme repercussions on pregnant women and their unborn children including increased maternal mortality and miscarriage rates $[5,6]$. Ebola virus is a disease in which fever and haemorrhage are caused by a viral infection, it is uncommon outside of Africa yet serious [7]. There have been over 28,618 reported cases, there have been numerous studies undertaken to measure the impact of the epidemic on the general population. However, only a small amount of research specifically focused on the impact of Ebola virus on pregnant women and their babies [5].

A study by Bebell, et al. [5], reported an $86 \%$ maternal mortality rate for the 111 reported cases of pregnant women with Ebola virus during the epidemic. This finding reinforces the extreme danger epidemics can place on pregnant women at high-risk. Further, Chertow, et al.'s [6] clinical observations of over 700 infected patients revealed that of the four pregnant women treated for the virus, three died soon after miscarrying and the one mother who survived also miscarried. Findings revealed that the mortality risk for both the mother and child was greater than for women who were not pregnant $[5,7]$. Knowledge of the increased mortality risk for pregnant women had the potential to spread fear and anxiety at the time, which according to Jamieson, et al. [7], contributed to reluctance to access antenatal services. Conversely, studies provided no evidence that pregnant women were more likely to contract the disease [5]. Furthermore, it is acknowledged that the true impact of Ebola virus on pregnant women is not completely understood due to the lack of data on pregnancy and maternal outcomes [5]. Of note, after examining the limited research available on the effect of Ebola virus during pregnancy, there is a significant focus on physical factors such as miscarriage and mortality rates as opposed to emotional, psychological or mental health factors. The limitation of research could be attributed to the concept that mental health conditions are underreported compared to physical health conditions due to stigma including issues with shame, guilt and self-esteem [8].

\section{Human Immunodeficiency Virus (HIV)}

Similar to Ebola virus, literature predominantly focuses on the physical implications of HIV on the health of pregnant women such as increased mortality rates due to anaemia and miscarriage [9]. For instance, Chertow, et al. [6] conducted clinical observations on over 700 infected patients between August and October 2014. However, studies undertaken by Kwalombota, et al. [10], and Ross, et al. [11], in Zambia and Thailand reported that a key adverse effect of HIV for pregnant women was the impact on mental health. Findings re- vealed women who were pregnant at the height of HIV experienced disbelief, fear, anxiety as well as depression and suicide $[10,11]$.

A study conducted in Zambia in the early years of HIV involved 45 pregnant women who were HIV-positive, assessed their psychological state [10]. The participants were placed into two groups, namely Group A included women who discovered they were HIV-positive during pregnancy, while Group B included women who were aware of their HIV-positive status before becoming pregnant. Through the use of a structured questionnaire, informal discussions were held, with results indicating women from both groups reported symptoms of depression, with $85 \%$ of the women claiming to have extreme depressive incidences and suicidal thoughts. The research highlighted that women in Group A were more susceptible to depressive episodes and had all considered terminating their pregnancy. In comparison, women in Group B who knew of their HIV status before failing pregnant reported lower rates of depression, anxiety and suicidal thoughts. Most noteworthy, 95\% of women from Group A and $60 \%$ from Group B reported they had lost interest in life and felt worthless. Furthermore, all women felt they were harming their babies due to their HIV status, a key finding that emphasises the psychological impact of HIV on a mother and her connection to her baby [10].

The connection between pandemics and depression and suicide was identified by Kwalombota in 2002 and later reinforced in a study by Ross, et al. in 2007 [11]. Ross, et al's., Qualitative phenomenological study included ten women who were HIV-positive from Thailand and examined their lived experience during pregnancy. Findings from multiple in-depth interviews that used open-ended questions reported that 'struggle' was the key theme to emerge. The women revealed they perceived their lives to be a struggle which Ross, et al. [11] conveyed using four subthemes that described their understanding of the various struggles the women experienced. For example, the women relayed they encountered struggling alone, struggling for the sake of the baby, struggling through ups and downs of living with HIV and sharing their struggle with others. Additionally, Ross, et al. [11] emphasised the greatest difficulty for the women was having to struggle alone during the early phase of their diagnosis. After being diagnosed with HIV, women experienced feelings of disbelief, fear, anxiety, and depression with half of the women admitting having contemplated suicide. Importantly, Kwalombota and Ross, et al. indicated the global HIV epidemic had a significant impact on the mental health and wellbeing of pregnant women.

\section{$\mathrm{H} 1 \mathrm{~N} 1$ influenza}

Another pandemic that impacted on pre and postnatal experiences of women was the H1N1 influenza in 2009. Commonly known as swine flu, H1N1 influenza was a new strain of the Influenza A virus that caused the spread of a highly infectious respiratory disease and was linked to higher mortality rates for pregnant women [3]. The first outbreak began in April 2009 and by September 2009 there was more than 296,471 confirmed cases globally, and 3486 deaths 
[12]. A case study by Hewagama, et al. [12] used data from six hospitals in Victoria, Australia to measure the impact of H1N1 influenza on pregnancy outcomes of patients admitted between May and July 2009. During the period of May to June, forty-three pregnant women were diagnosed with H1N1 influenza. Of these cases, eight involved admission to intensive care, with two fetal deaths, one maternal death and one neonatal death. Study results confirmed H1N1 influenza caused pregnant women to experience a high number of complications however, the study exclusively reported on the physical health outcomes of pregnant women with H1N1 influenza and the impact of the disease on mental health was not evident.

Hewagama, et al's., [12] results correlate with the findings of Mosby, et al. [13]. Mosby, et al. reviewed 2153 abstracts and selected 120 studies to determine the physical outcomes for pregnant women infected with H1N1 influenza [13]. The data focused on risk factors, treatment and number of cases. Similarly, the review determined that pregnant women with H1N1 influenza had an increase probability of hospitalisation, admission to intensive care and death [13]. Again, the focus of the study was physical health outcomes and lacked inclusion of the impact on mental health. Both Hewagama, et al. [12] and Mosby, et al. [13] determined that the physical health experiences of pregnant women during the pandemic were seriously altered due to the high physical health risks they faced. However, literature examining the mental health impacts on pregnant women during $\mathrm{H} 1 \mathrm{~N} 1$ remains limited, therefore, it is difficult to determine the full mental health impact of the pandemic on pregnant women. As mentioned previously, this limitation could be attributed to the concept that mental health conditions were not addressed or discussed due to the stigma of mental illness and the associated shame, guilt and lowered self-esteem [8].

Overall, findings surrounding the history of key epidemics and pandemics indicated that pregnant women experienced increased physical health risks. However, there is limited research and findings to indicate whether epidemics and pandemics have an impact on the mental health of pregnant women. As such, the varied mental health effects and outcomes as well as the potential long-term health implications of pandemics and epidemics are not fully understood and need to be a priority for future research.

\section{Expected Psychological Adjustments to Preg- nancy}

This exploration of pre and postnatal experiences of pregnant women during COVID-19 would be incomplete without understanding the psychological adjustments women typically experience throughout pregnancy. The following section will explore the literature surrounding the key psychological adjustments experienced in pregnancy, to provide context to the research of pre and postnatal experiences during COVID-19.

Almost all systems in a woman's body undergo requisite changes after becoming pregnant to allow for maternal adaptation and development of a healthy fetus [14]. Most sig- nificantly, changes occur to the psychological functioning of a pregnant woman from the start of pregnancy through to the postpartum period [15]. Pregnant women experience psychological adjustments to a varied degree, and it is expected that a pregnant woman will experience psychological ambivalence, mood swings such as fatigue to elation, emotional disturbances, anxiety and depressive states [14-16]. Further, it is relevant that this paper examines psychological adjustments such as the level of anxiety and ambivalence experienced by pregnant women, as anxiety and ambivalence is the predominant adjustments experienced [15-21].

Feelings of uncertainty and anxiety are usual and anticipated psychological adjustments aligned with pregnancy $[15,18,19,21]$. For instance, Malhotra, et al. (2015) [18] studied the nature and prevalence of happiness among 100 pregnant women in India and identified that in the first trimester it is common for pregnant women to experience nervousness and anxiety due to fear of losing the baby. Malhotra used the qualitative approach of grounded theory which included semi-structured interviews, to provide comprehensive insight into the lived experiences of the participants to identify predominant themes. Similarly, Schneider's [19] qualitative study explored the experience of 13 Australian women's first pregnancy and determined that the majority of women found it difficult to cope with the emotional symptoms, with loss of control being a common cause of anxiety throughout pregnancy. Furthermore, Soltani, et al.'s [21] qualitative study of 19 healthy pregnant women in Iran explored the perceived challenges of adapting to a first pregnancy during the third trimester determined that women experienced fear and worry. Thus, Malhotra, Schneider, Soltani, et al. and Bjelica, et al. identified that the key psychological challenges pregnant women experienced included anxiety, uncertainty and worry aligned with becoming a mother and accepting the associated responsibilities of motherhood.

Ambivalence is also recognised as a common psychological adjustment experienced during pregnancy $[16,17,20]$. For example, Lederman and Weis's, et al. [16] research on the psychosocial adaptation to pregnancy recognised that ambivalence in pregnancy can be experienced by women in the initial stages of pregnancy and throughout. Similarly, Shahoei, et al.'s [20] qualitative study that explored the experiences of 22 Kurdish pregnant women in the third trimester concluded that the women experienced phases of ambivalence with many contradicting feelings. Furthermore, Shahoei, et al. [20] determined that women felt unpleasant and joyous, happy and troubled and enjoyed the experience of pregnancy and birth but was also afraid of birth. It was noted that a potential limitation to Shahoei, et al.'s [20] study was that responses may have differed due to women being from different cultures. However, research of 84 pregnant American women by Cutler, et al. [17] supported Lederman and Weis's and Shahoei, et al.'s research highlighting that ambivalence is an expected occurrence in pregnancy, but ambivalence doesn't need to signify a negative experience or rejection of pregnancy. Thus, the literature highlights the understanding that ambivalence is an anticipated adjustment in pregnancy but heightened ambivalence can impact on the mental health of 
Citation: Barnes A, Kelly J (2021) Antenatal and Postnatal Experiences of Women during Covid-19 Pandemic: The Past Meets the Present. J Nurs Pract 4(1):302-308

pregnant women in some cases $[16,20]$.

The psychological adjustments experienced during pregnancy confirms that anxiety and ambivalence are commonly experienced and considered to be a normal adjustment in a healthy pregnancy [15-21]. Feelings of anxiety and ambivalence although normal and expected in pregnancy, when matched with a pandemic have the potential to impact upon the mental health and wellbeing of the women who are pregnant, therefore warranting exploration into the experiences of women who are pregnant during the COVID-19 pandemic.

\section{Coronavirus Disease 19}

The Coronavirus disease 19 (COVID-19) is a viral infection caused by severe acute respiratory syndrome coronavirus 2 (SARS-CoV-2) and is extremely infectious, with more than seventy thousand people infected and over eighteen hundred people killed within the first fifty days of the epidemic [2]. As the number of infections and deaths increased rapidly across the globe, combined with the lack of human immunity, risk of transmission from asymptomatic carriers and unconfirmed incubation period, the World Health Organisation declared COVID-19 a pandemic on the 11 March 2020 [22,23]. Therefore, the following section addresses the transmission, symptoms, treatment and the Australian context and public health response to COVID-19, to provide context for the exploration into the pre and postnatal experiences of pregnant women during the pandemic.

The symptoms of COVID-19 usually present within two to 14 days and vary in severity, from asymptomatic or mild common cold or flu symptoms to major symptoms including chest pain, breathing issues, confusion, cyanosis of the face and lips and pneumonia [2,23]. Intensive care unit admission with specialised respiratory management is mostly required for patients presenting with persistent, major symptoms. Cortegiani, et al. [24] reported $15 \%$ of people suffer severe symptoms however, Additionally, Lal, et al. [23] highlighted that adults and the elderly are at greater risk of suffering severe physical symptoms than children.

Currently, there is no proven effective treatment specifically for COVID-19. Medical care involves responding to and treating symptoms in attempt to reduce the viral load [2]. The current approach of managing symptoms has been employed whilst authorised vaccines become available on a global scale. Although a number of vaccines have been approved for human use and rollout programs have commenced in different countries, until approved and diversified vaccinations are available globally control of the pandemic will not be achievable and the covid-19 pandemic will continue $[2,25]$.

\section{COVID-19 in Australia}

Australia's first confirmed case of COVID-19 acquired overseas, was reported on January $25^{\text {th }} 2020$ in Melbourne, Victoria. The first community acquired or localised case occurred in Queensland in the first week of February (Fitzgerald \& Wong, 2020) [22]. Fitzgerald and Wong [22] and Higginson, et al. [26] noted the Australian government reacted early to the pandemic and implemented a consolidated approach across the country in an attempt to reduce the risk of transmission.

The consolidated approach adopted by Australia was based on four key strategies including State border closures, wide-spread testing, contact tracing, physical distancing principles and personal hygiene practices [22]. Each State in Australia developed a set of measures using the four key strategies that mostly included, local, domestic and international travel bans, home schooling, working from home, social distancing rules, public health campaigns, mass quarantines, cluster lockdowns and temporary closure of non-essential businesses and industries including sport [22,26]. Higginson, et al.'s mixed method study on the economic impact of COVID-19 on health care policy decisions, reported that Australia's strong healthcare system enabled timely and strict measures to be introduced. Additionally, State legislation enforced jurisdiction to penalise any non-adherence to the imposed safety measures.

Studies conducted by Fitzgerald and Wong, et al. [22] and Higginson, et al. [26] demonstrate that the enormous, long term impacts of COVID-19 and effect of imposed strategies on the economy, employment, community, education and mental and physical health outcomes, are not fully recognised nor understood. Research is urgently needed to fully understand the impact of this pandemic thus, highlighting the importance of qualitative research into the pre and postnatal experiences of pregnant women during COVID-19.

\section{The Psychological Impact of COVID-19 and Restrictions}

In order to slow the spread of COVID-19 and reduce transmission in Australia, a series of lockdown measures were introduced nationally, they varied in each state but mostly included, lockdowns, home schooling, working from home, social distancing rules and mass quarantines [22,26]. Preliminary studies have determined a direct link between the imposed lockdown measures, COVID-19 and declining mental health [27-29]. As such, the combination of the mental health of individuals, lockdown measures and the physical health risks COVID-19 require further investigation and emphasises the need to explore the impact of COVID-19 on pre and postnatal women [23,27-29].

In 2020, Brooks, et al. conducted a review surrounding the impact of quarantine indifferent countries and different pandemics. Twenty-four studies involving data from H1N1 and Ebola were examined and findings determined that quarantine measures have a negative psychological impact causing anger, confusion and symptoms of post-traumatic stress. Further, Burhamah, et al.'s [28] quantitative study involving over 4000 participants from Kuwait, used an online survey to determine more specifically the impact COVID-19 lockdown measures had upon mental health. Similar to Xiong, et al. (2020) [29], Burhamah, et al. [28] found that women had a higher tendency for depression and anxiety. Furthermore, both studies concluded that COVID-19 lockdown measures and quarantine cause a substantial burden on mental health, which linked with the overall psychological impact of COV- 
ID-19 established by Xiong, et al. [29], highlights the importance of further research into the mental health of pre and postnatal women during the COVID-19 pandemic. Additionally, Xiong, et al. [29] and Burhamah, et al. [28] emphasise that focusing on mental health during COVID-19 is an international health priority, in which prevention, accessible support and awareness need to be a priority.

Further, a systematic review involving 19 studies was conducted by Xiong, et al. [29] examined the impact of COVID-19 pandemic on the mental health status of the public. Xiong, et al.'s [29] review supports the findings of both Brooks, et al.'s [27] and Burhamah, et al.'s [28] study into the impact of lockdown and quarantine on mental health. The review included studies from eight countries, which although limited determined the impact at a global level, the variation and inclusion of high, middle and low income provided a diverse perspective. Xiong, et al. [29] determined a higher prevalence of mental health symptoms amongst the public post COVID-19, bringing to attention the significantly higher levels of psychological distress many are experience as a result of COVID-19. The review also determined that women were more likely to develop depressive symptoms compared to men, a significant finding when exploring the potential impact of COVID-19 on the experiences of pre and postnatal women.

\section{COVID-19 and Pregnancy}

Research regarding the ongoing COVID-19 pandemic and the impact on women who are pregnant and give birth is still emerging. However, from the limited literature available researchers are in agreement that there are four key are as requiring investigation into the experiences of women who are pregnant and give birth during the COVID-19 pandemic. These areas include, vulnerability to contracting COVID-19, impact on mental health outcomes, impact of health safety measures and the need for further research into this phenomenon [30-36].

The female body adapts both immunologically and physically during pregnancy. Therefore, women who are pregnant have a reduced immune response and are more prone to infection [14,30,34]. Ali \& Shahil Feroz and Matvienko-Sikar, et al. also highlighted that women who are pregnant are more vulnerable to contracting viral infections such as COVID-19. The increased risk is linked to adverse physical health outcomes and adverse mental health outcomes [30,34]. Further, antenatal and postnatal periods are a pivotal, transitional time combined with an increased vulnerability of being immune-compromised [34]. Therefore, during a global pandemic, there exists an increased potential for COVID-19 to effect the physical and psychosocial experiences of women who are pregnant and give birth during this time [30,31,34].

Examining the impact of COVID-19 on symptoms of anxiety and depression among pregnant women has commenced. For example, a study conducted in China by $\mathrm{Wu}$, et al. [36] and a study in Italy by Saccone, et al. [35] has investigated psychological responses of pregnant women during the COVID-19 pandemic. Further Corbett, et al. [32] determined the prevalence of maternal anxiety due to COVID-19 and Dav- enport, et al. [33] assessed the impact of COVID-19 and subsequent health measures on the mental health of pregnant and postpartum women. Thus, since the onset of COVID-19 research has been undertaken which has identified a link between the pandemic and a rise in adverse mental health outcomes. More specifically, Wu, et al.'s (2020) [36] cross-sectional study involved Chinese women in their third trimester and the use of the self-reporting tool the Edinburgh Postnatal Depression Scale. The study compared the mental state of 2839 participants measured prior to COVID-19 to the mental state of 1285 participants measured after the outbreak was declared in China. Findings revealed there was an increased rate of depressive and anxiety symptoms including self-harm amongst the pregnant women assessed after the outbreak, thus confirming the link between COVID-19 and adverse mental health outcomes [36].

The connection between COVID-19 and adverse mental health outcomes for pregnant women was also significant in Saccone, et al.'s [35] cross-sectional survey on the psychological impact of COVID-19 and levels of anxiety in pregnant women. Findings by Saccone, et al. [35] revealed COVID-19 had a moderate to severe impact on the participants psychological health, and mental health of pregnant women during the pandemic. Of note, of the 100 women who participated in the study, none had a prior diagnosis of mood disorders or post-natal depression in previous pregnancies. Another relevant study conducted post COVID-19 was undertaken by Davenport, et al. [33], Davenport, et al. assessed the impact of COVID-19, poor psychological health and subsequent health measures on the mental health of 520 pregnant and 380 women within the first year after giving birth.

Davenport, et al. [33] identified a substantial increase in anxiety and depression levels amongst the study participants, further highlighting the connection between COVID-19 and poor mental health outcomes for pregnant and postpartum women $[32,33,35]$. Furthermore, the need for further investigation into the impact and adverse outcomes is required [33]. Both Davenport, et al. [33] and Wu, et al. [36] used the Edinburgh Postnatal Depression Scale, a self-reported questionnaire that although an internationally recognised and recommended screening tool it was noted as a potential limitation in the studies due to not having the ability to provide a diagnosis. Also, whilst Davenport, et al. [33] only captured one demographic of women from Canada, the results of this study paired with the results of studies from China and Italy provide a comprehensive and diverse range of findings and emphasise the impact of COVID-19 on the experiences of women who are pregnant and give birth during the pandemic.

Health measures introduced to reduce the transmission of COVID-19 have also been identified as having an adverse impact upon the mental health of pre and postnatal women $[30,31,34]$. For instance, lockdown measures and quarantine implemented to manage COVID-19, have caused a substantial burden on mental health in the general population [2729]. The potential of social distancing measures impacting on mental health combined with the proven increased risk of mental health issues pregnant and postnatal women face during COVID-19, emphasises the need to undertake research 
on pre and postnatal women. Measures such as mass lockdowns, social distancing and travel bans have the potential to increase anxiety and depressive symptoms, increase the demand on health care services, and lead to additional concerns for pre and postnatal women $[30,34]$.

The potential impact of COVID-19 on the experiences of women who are pregnant and give birth during the pandemic requires further investigation $[32,33,36]$. Although Ali \& Shahil Feroz [30] acknowledge that the consequences of the outbreak are still emerging, further research should be noted as an area of priority. The need for further investigation into the impact of health safety measures and extreme changes to routine and support for women who are pregnant and give birth has been presented in this paper. Finally, as emphasised by Saccone, et al., [35] Buekens, et al. [31], Xiong, et al. [29] and Burhamah, et al. [28] mental health during COVID-19 is an international health priority.

\section{Summary}

Historically, epidemics and pandemics have had significant impact on the health of individuals but most noteworthy is the impact on the physical and psychological health of women who are pregnant. The latest pandemic, COVID-19 will have a long-term impact in terms of health and wellbeing as a result of the infection but also the measures imposed to reduce transmission. At present, research is starting to emerge regarding the impact of COVID-19 on the mental health of women who were pregnant and give during the pandemic. However, there is limited literature and limited qualitative research that has focused on the antenatal and postnatal women in terms of physical and mental health issues. Recent studies have concluded that mechanisms to control COVID-19 such as lockdown measures and quarantine have led to a substantial burden on mental health. The need for further research and the mental health of individuals during COVID-19 is an international health priority $[28,29]$. Therefore, this paper illustrates the significance of future research such as a qualitative study into the pre and postnatal experiences of pregnant women during the COVID-19 pandemic.

\section{Acknowledgements}

The authors would like to acknowledge that there has been no financial assistance with the project. The authors acknowledge the support of RMIT University to complete this paper.

\section{References}

1. Dyer O (2021) Covid-19: WHO says laboratory escape theory is "extremely unlikely" after mission to China. BMJ, 372.

2. Shereen MA, Khan S, Kazmi A, et al. (2020) COVID-19 infection: Origin, transmission, and characteristics of human coronaviruses. Journal of Advanced Research 24: 91-98.

3. Clements BW, Casani JAP (2016) 16 - Pandemic influenza. Disasters and Public Health 385-410.

4. Potter CW, Jennings R (2011) A definition for influenza pandemics based on historial records. Journal of Infection 63: 252-259.

5. Bebell LM, Oduyebo T, Riley LE (2017) Ebola virus disease and pregnancy: A review of the current knowledge of Ebola virus pathogenesis, maternal, and neonatal outcomes. Birth Defects Research 109: 353-362.

6. Chertow DS, Kleine C, Edwards JK, et al. (2014) Ebola virus disease in West Africa - Clinical manifestations and management. New England Journal of Medicine 371: 2054-2057.

7. Jamieson DJ, Uyeki TM, Callaghan WM, et al. (2014) What obstetrician-gynecologists should know about Ebola: A perspective from the Centers for Disease Control and Prevention. Obstetrics \& Gynecology 124: 1005-1010.

8. Bharadwaj P, Pai MM, Suziedelyte A (2017) Mental health stigma. Economics Letters 159: 57-60.

9. McIntyre J (2005) Maternal health and HIV. Reproductive Health Matters 13: 129-135.

10. Kwalombota M (2002) The effect of pregnancy in HIV-infected women. AIDS Care 14: 431-433.

11. Ross R, Sawatphanit W, Draucker CB, et al. (2007) The lived experiences of HIV-positive, pregnant women in Thailand. Health Care for Women International 28: 731-744.

12. Hewagama S, Walker SP, Stuart RL, et al. (2010) 2009 H1N1 Influenza A and pregnancy outcomes in Victoria, Australia. Clinical Infectious Diseases 50: 686-690.

13. Mosby LG, Rasmussen SA, Jamieson DJ (2011) 2009 pandemic influenza $A$ (H1N1) in pregnancy: A systematic review of the literature. American Journal of Obstetrics and Gynecology 205: 10-18.

14. Jordan RG, Engstrom J, Marfell J, et al. (2013) Prenatal and Postnatal Care. John Wiley \& Sons, Incorporated.

15. Bjelica A, Cetkovic N, Trninic-Pjevic A, et al. (2018) The phenomenon of pregnancy - A psychological view. Ginekol Pol 89: 102106.

16. Lederman R, Weis K (2009) Psychosocial adaptation to pregnancy: Seven dimensions of maternal role development. Springer New York, USA.

17. Cutler A, McNamara B, Qasba N, et al. (2018) "I Just Don't Know": An exploration of Women's ambivalence about a new pregnancy. Women's Health Issues 28: 75-81.

18. Malhotra R, Mudgal R, Dharmarha S, et al. (2015) How happy are pregnant women?: A socio-demographic analysis. Clinical Epidemiology and Global Health 3: 117-124.

19. Schneider $Z$ (2002) An Australian study of women's experiences of their first pregnancy. Midwifery 18: 238-249.

20. Shahoei R, Riji HM, Saeedi ZA (2011) Kurdish pregnant women's feelings: A qualitative study. Midwifery 27: 215-220.

21. Soltani F, Maleki A, Shobeiri F, et al. (2017) The limbo of motherhood: Women's experiences of major challenges to cope with the first pregnancy. Midwifery 55: 38-44.

22. Fitzgerald DA, Wong GWK (2020) COVID-19: A tale of two pandemics across the Asia Pacific region. Paediatric Respiratory Reviews 35: 75-80.

23. Lal P, Kumar A, Kumar S, et al. (2020) The dark cloud with a silver lining: Assessing the impact of the SARS COVID-19 pandemic on the global environment. Science of the Total Environment 732: 139297.

24. Cortegiani A, Ingoglia G, Ippolito M, et al. (2020) A systematic review on the efficacy and safety of chloroquine for the treatment of COVID-19. Journal of Critical Care 57: 279-283. 
25. Wouters O, Shadlen K, Salcher-Konrad M, et al. (2021) Challenges in ensuring global access to COVID-19 vaccines: Production, affordability, allocation, and deployment. The Lancet 397: 10231034.

26. Higginson S, Milovanovic K, Gillespie J, et al. (2020) COVID-19: The need for an Australian economic pandemic response plan. Health Policy and Technology 9: 488-502.

27. Brooks SK, Webster RK, Smith LE, et al. (2020) The psychological impact of quarantine and how to reduce it: Rapid review of the evidence. The Lancet 395: 912-920.

28. Burhamah W, AlKhayyat A, Oroszlányová M, et al. (2020) The psychological burden of the COVID-19 pandemic and associated lockdown measures: Experience from 4000 participants. Journal of Affective Disorders 277: 977-985.

29. Xiong J, Lipsitz O, Nasri F, et al. (2020) Impact of COVID-19 pandemic on mental health in the general population: A systematic review. Journal of Affective Disorders 277: 55-64.

30. Ali NA, Shahil Feroz A (2020) Maternal mental health amidst the COVID-19 pandemic. Asian Journal of Psychiatry 54: 102261.
31. Buekens P, Alger J, Bréart G, et al. (2020) A call for action for COVID-19 surveillance and research during pregnancy. The Lancet Global Health 8: e877-e878.

32. Corbett GA, Milne SJ, Hehir MP, et al. (2020) Health anxiety and behavioural changes of pregnant women during the COVID-19 pandemic. European Journal of Obstetrics \& Gynecology and Reproductive Biology 249: 96-97.

33. Davenport MH, Meyer S, Meah VL, et al. (2020) Moms are not ok: COVID-19 and Maternal Mental Health. Frontiers in Global Women's Health 1.

34. Matvienko-Sikar K, Meedya S, Ravaldi C (2020) Perinatal mental health during the COVID-19 pandemic. Women and Birth 33: 309-310.

35. Saccone G, Florio A, Aiello F, et al. (2020) Psychological impact of coronavirus disease 2019 in pregnant women. American Journal of Obstetrics and Gynecology 223: 293-295.

36. Wu Y, Zhang C, Liu H, et al. (2020) Perinatal depressive and anxiety symptoms of pregnant women during the coronavirus disease 2019 outbreak in China. American Journal of Obstetrics and Gynecology 223: 240.E1-249.E9.

DOI: $10.36959 / 545 / 398$

Copyright: (c) 2021 Barnes A, et al. This is an open-access article distributed under the terms of the Creative Commons Attribution License, which permits unrestricted use, distribution, and reproduction in any medium, provided the original author and source are credited. 